\title{
Editorial
}

\section{Red Académica Asesora de Revisiones Sistemáticas (RAARS)}

\author{
Academic Advisory Network for Systematic Reviews (AANSR)
}

\author{
Víctor Manuel Mendoza-Núñez ${ }^{1}$ \\ ${ }^{1}$ Editor en Jefe de la Revista Casos y Revisiones de Salud
}

\section{INTRODUCCIÓN}

Las revisiones sistemáticas (RS), tal como las definen Ferreira González et al. (2011), son "investigaciones científicas en las cuales la unidad de análisis son los estudios originales primarios. Constituyen una herramienta esencial para sintetizar la información científica disponible, incrementar la validez de las conclusiones de estudios individuales e identificar áreas de incertidumbre donde sea necesario realizar investigación. Además, son imprescindibles para la práctica de una medicina basada en la evidencia y una herramienta fundamental en la toma de decisiones médicas". ${ }^{1}$ Por tal motivo, su metodología debe rigurosa, para que pueda ser confiable y reproducible por otros investigadores y clínicos interesados en la aplicación del conocimiento científicamente comprobado, ya que a través de una RS se puede disponer de una síntesis de los hallazgos de los estudios metodológicamente confiables. Para tal efecto, a nivel internacional se han establecido los lineamientos para que las RS cumplan con dicho objetivo. En este sentido, las RS deben seguir con los criterios de la declaración de PRISMA (por su siglas en inglés, Preferred Reporting Items for Systematic reviews and Meta-Analyses). ${ }^{2}$ En este sentido, PRISMA constituye una guía estructurada para la búsqueda y análisis de la in-

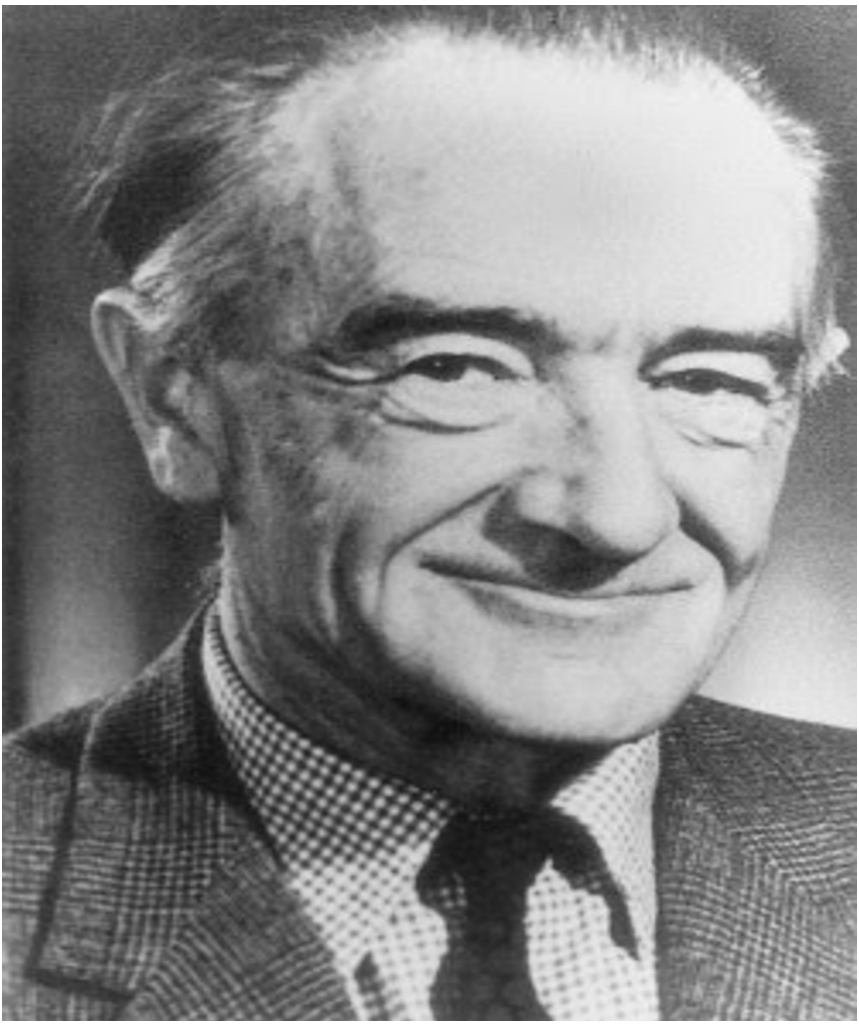

Figura 1. Archibald Leman Cochrane (1909-1988). Reconocido como el padre la Medicina Basada en la Evidencia. ${ }^{4}$

Mendoza-Núñez VM. Red Académica Asesora de Revisiones Sistemáticas (RAARS). CyRS. 2021; 3(2):5-13. DOI: https://doi.org/10.22201/fesz.26831422e.2021.3.2.1 
formación científica disponible, para la elaboración de una síntesis de los resultados de un tema específico, con el propósito de toma de decisiones clínicas y de salud pública fundamentadas. Al respecto, entre los pioneros e impulsores de la metodología para las RS destaca Archibald Leman Cochrane (1909-1988) (Figura 1), médico escocés, quien en 1979 publicó el libro "Effectiveness and Efficiency: Random Reflections on Health Services", en el que resalta la importancia de la metodología rigurosa de la investigación clínica a través del desarrollo de ensayos clínicos controlados aleatorizados, para hacer que la medicina más efectiva y eficiente. ${ }^{2}$ Por tal motivo, Cochrane es reconocido como uno de los padres de la epidemiología clínica moderna y la medicina basada en la evidencia (MBE). En este sentido, en 1992 se fundó el "Centro Cochrane" en Oxford, Reino Unido y en 1993 surgió la "Colaboración Cochrane" con la participación de 11 países y actualmente participan 130 países y con alrededor de 37,000 colaboradores. . $^{4-6}$

La relevancia y aplicación de la metodología de PRISMA para las RS no se ha limitado a la medicina, si no que ha trascendido a los campos de estudios de la biología, enfermería, odontología, nutriología, psicología, ciencias sociales e incluso se sugiere su aplicación en la ingeniería química. ${ }^{7-13}$

En este contexto, además de la publicación de artículos científicos, la metodología PRISMA puede ser utilizada para la elaboración de tesis de licenciatura y posgrado. Sin embargo, en la Facultad de Estudios Superiores Zaragoza, UNAM, (FES Zaragoza), son pocos los profesores e investigadores que tienen el conocimiento y experiencia en la aplicación de dicha metodología para la asesoría de tesis, de ahí la importancia de proponer y desarrollar estrategias que permitan ampliar la formación de profesores e investigadores, para que funjan como asesores de tesis de pregrado y posgrado de estudios de revisiones sistemáticas, para lo cual consideramos que una de los mejores opciones es la conformación de una Red Académica Asesora.

\section{Antecedentes}

La FES Zaragoza desde hace más de dos décadas, ha impulsado la formación de recursos humanos sobre metodología de la investigación científica en el campo de la salud, a través de la impartición del Diplomado en Investigación Clínica y Epidemiológica (DICE). En este sentido, han egresado 28 generaciones del DICE (alrededor de 600), contribuyendo en el desarrollo de la investigación científica clínica, epidemiológica y de salud pública en la FES Zaragoza y en el sector salud a nivel nacional. El grupo de profesores-investigadores del DICE han publicado algunos textos con el propósito de hacer accesible el marco conceptual y metodológico para la elaboración de proyectos de investigación científica en el ámbito de la salud.

- Manual para la elaboración de proyectos de investigación en ciencias de la salud. ${ }^{14}$

- Investigación. Introducción a la metodología. ${ }^{15}$

- Análisis y difusión de resultados científicos. ${ }^{16}$

- Metodología de la investigación. Un enfoque práctico. ${ }^{17}$

Al respecto, dichos textos constituyen un material didáctico que ha contribuido en la formación académica de los alumnos de pregrado y posgrado de la FES Zaragoza, incluso han sido incluidos como bibliografía de los programas sintéticos de los Planes de Estudios de las carreras de Cirujano Dentista, Médico Cirujano, Enfermería y QFB, y en los programas de las especialidades de Estomatología en Atención Primaria, Estomatología del Niño y de Adolescente. También son consultados por alumnos de otras universidades del país.

Por otro lado, en 2018 el Comité de Investigación de la FES Zaragoza, aprobó la creación del Programa para la Investigación Bibliográfica Científica sobre Salud (PIBCIS), con el propósito de formar grupos académicos que promuevan la investigación bibliográfica científica sobre salud, para desarrollar una cultura académica sustentada en la metodología internacionalmente aceptada para la revisión y elaboración de escritos científicos. Asimismo, en el marco del PIBCIS, se planeó la edición de la Revista Casos y Revisiones de Salud, cuyo primer número fue publicado en julio de 2018. También se propuso la creación de una red académica, para impulsar la formación de recursos huma- 
nos para la aplicación y asesoría de la metodológica para la elaboración de artículos científicos y tesis con la metodología de PRISMA para RS.

\section{Red Académica Asesora de Revisiones Sistemáticas (RAARS)}

El 15 de octubre de 2021, se conformó formalmente la Red Académica Asesora de Revisiones Sistemáticas (RAARS) y se presentó su página Web (https://www.raars.zaragoza.unam.mx/) (Figuras 2-4), ${ }^{18}$ cuyo medio de comunicación permitirá promover la formación académica de profesores y es-

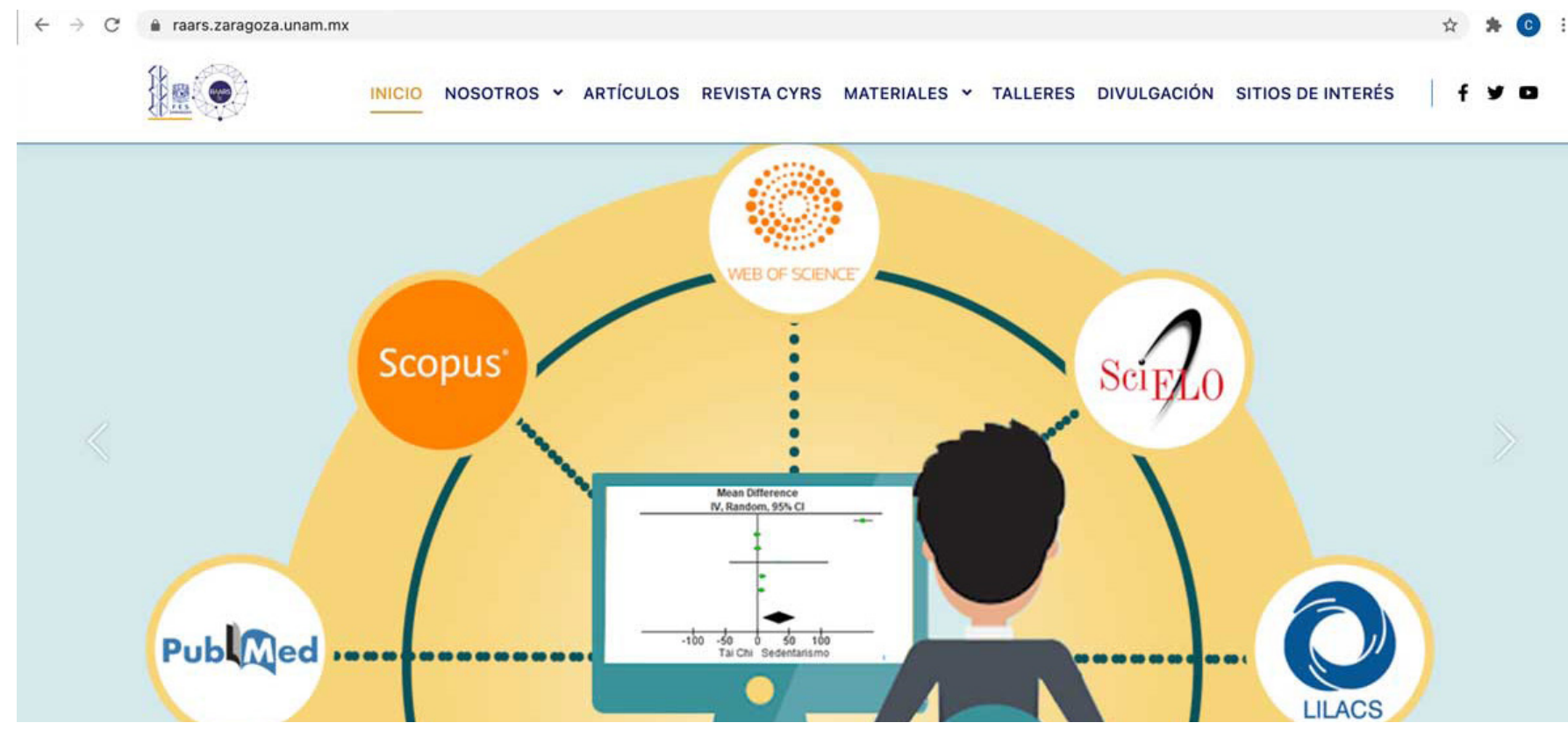

Figura 2. Página web de la RAAS

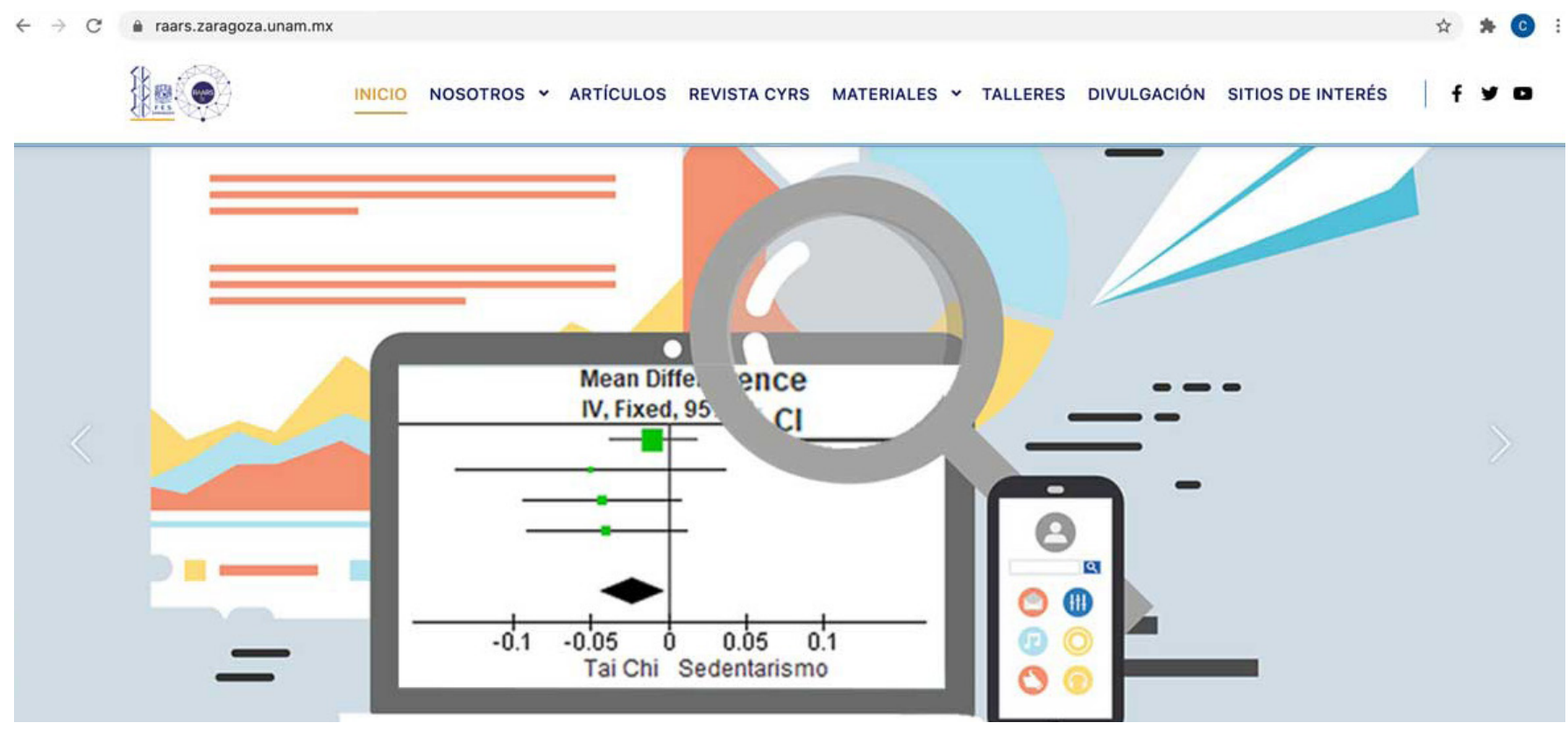

Figura 3. Página web de la RAAS 
$\leftarrow \rightarrow C$ raars.zaragoza.unam.mx

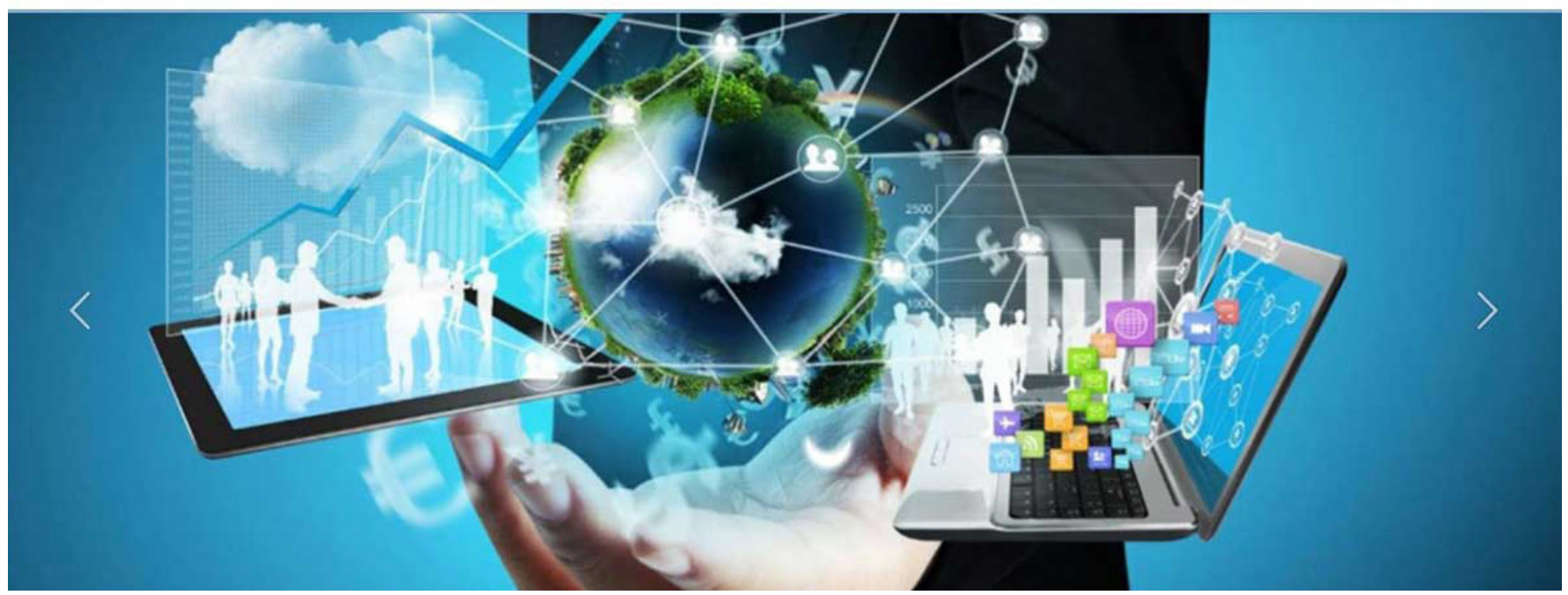

Figura 4. Página web de la RAARS

tudiantes de pregrado y posgrado sobre la metodología de la investigación para llevar a cabo estudios con la metodología PRISMA para revisiones sistemáticas y meta-análisis. La RAARS, constituye una estrategia para la formación académica y mejoramiento de la enseñanza, como parte del proyecto PAPIME PE203421 "Programa para la enseñanza a distancia de la metodología de revisiones sistemáticas para la elaboración de tesis de grado de licenciatura en la FES Zaragoza", aprobado por la Dirección General de Asuntos del Personal Académico de la UNAM, en la convocatoria 2021 del Programa de Apoyo a Proyectos para Innovar y Mejorar la Educación (PAPIME). El propósito de la RAARS es formar grupos académicos asesores para el desarrollo la investigación bibliográfica científica con la metodología de revisión sistemática y meta-análisis, para la elaboración de tesis de grado y manuscritos científicos, para su publicación en revistas especializadas. La RAARS establece como estrategia la vinculación profesores-investigadores de la FES Zaragoza con experiencia en investigación científica y con la metodología de PRISMA, con docentes que fungen como asesores de tesis de las carreras de Biología, Cirujano Dentista, Enfermería, Médico Cirujano, Nutriología, Psicología y Química Farmacéutico Biológica, interesados en aplicar la metodología de PRISMA, para la dirección y asesoría de tesis.

\section{FUNCIONES DE LA RAARS}

1. Formar a profesores de licenciatura sobre la metodología de revisiones sistemáticas y metaanálisis acorde con los lineamientos de PRISMA, para la asesoría de tesis de licenciatura de las carreras de Biología, Cirujano Dentista, Enfermería, Médico Cirujano, Química Farmacéutico Biológica, Nutriología y Psicología de la FES Zaragoza, UNAM.

2. Apoyar metodológicamente a los profesores de las carreras antes mencionadas durante la asesoría de las tesis.

3. Asesorar metodológicamente a los profesores de las carreras antes señaladas para la elaboración de manuscritos para su posible publicación en revistas nacionales e internacionales.

4. Organizar cursos y talleres sobre la aplicación de la metodología de revisiones sistemáticas y meta-análisis para profesores y estudiantes de pregrado y posgrado.

5. Elaborar material didáctico sobre aspectos conceptuales de las revisiones sistemáticas y metaanálisis. 
6. Promover las buenas prácticas de la metodología de la investigación.

7.Difundir las ligas de interés sobre metodología de la investigación.

8. Contribuir con los objetivos y metas del Programa para la Investigación Bibliográfica Científica sobre Salud de la FES Zaragoza, UNAM (PIBCIS).

9. Difundir las tesis de revisiones y sistemáticas asesoradas por la RAARS.

10. Difundir los artículos de revisiones sistemáticas asesorados por la RAARS.

11. Difundir los artículos y boletines publicados en la Revista Casos y Revisiones de Salud.

\section{Requisitos de INGReso a LA RAARS}

\section{Ser profesor de la FES Zaragoza, UNAM.}

2. Haber asesorado tesis de licenciatura o haber cursado un posgrado en el que elaboró una tesis para su titulación.

3. Aprobar el taller "Diagnóstico sobre conocimientos de la metodología de revisiones sistemáticas y meta-análisis" organizado anualmente por la RAAS.

4. Aprobar el taller "Formación de profesores como asesores de tesis con la metodología de revisiones sistemática y meta-análisis (PRISMA)".

5. Tener interés para asesorar tesis de licenciatura o posgrado con la metodología PRISMA.

6. Tener un candidato(a) para realizar una tesis de licenciatura o posgrado.

7. Asesorar una tesis de licenciatura o posgrado con la metodología PRISMA

8. Solicitar el ingreso a la RAARS.

9. Cumplir con las funciones de la RAARS.

\section{INTEGRANTES DE LA RAARS}

\section{Consejo Académico y de Difusión}

\author{
Dr. Víctor Manuel Mendoza Núñez \\ Profesor de Posgrado \\ Prof. Carrera Titular C, TC definitivo \\ mendovic@unam.mx
}

\section{Dra. Martha Asunción Sánchez Rodríguez}

Profesora de la carrera de Química Farmacéutico Biológica

Prof. Carrera Titular C, TC definitiva

masanrod@comunidad.unam.mx

\author{
Dr. Osvaldo Daniel Castelán Martínez \\ Profesor de la carrera de Química Farmacéutico \\ Biológica \\ Prof. Carrera Asociado C, TC \\ castelan@unam.mx
}

\section{Mtra. Catalina Armendáriz Beltrán}

Profesora de la carrera de Ciencias de la Comunicación

Prof. Asignatura A

catalina.armendariz@gmail.com

Carrera de Biología

Dr. Edelmiro Santiago Osorio

Profesor de la carrera de Biología y del posgrado Ciencias Biológicas

Prof. Carrera Titular C, TC definitivo

edelmiro@unam.mx

\section{Dra. Graciela Gavia García}

Estancia Posdoctoral

graciela.gavia.garcía@gmail.com

Carrera de Cirujano Dentista

Dra. Rosa Diana Hernández Palacios

Profesora de la carrera de Cirujano Dentista

Prof. Carrera Titular A, TC Def

palaciosd671@gmail.com

Dra. Beatriz Hernández Monjaraz

Profesora de la carrera de Cirujano Dentista

Prof. Carrera Asociado C, TC

bety.labo@gmail.com 
Mtra. María Georgina López Jiménez

Profesora de la carrera de Cirujano Dentista y la Especialidad en Estomatología del Niño y el Adolescente Prof. Asignatura A

mtra.geo.lj@gmail.com

\section{Mtra. Cecilia Mecalco Herrera}

Profesora de la carrera de Cirujano Dentista

Prof. Carrera Asociado C, TC

mecalcocecilia@gmail.com

Carrera de Enfermería

\section{Mtra. Elsa Correa Muñoz}

Profesora de la Especialidad en Estomatología del Niño y del Adolescente y de la Maestría en Enfermería

Prof. Carrera Titular A, TC definitiva

elcommunam@gmail.com

Mtra. Cristina Flores Bello

Profesora de la carrera de Enfermería

Prof. Carrera Asociado B, TC

rasguosaflores@yahoo.com.mx

Carrera de Médico Cirujano

Dra. Itzen Aguiñiga Sánchez

Profesora de la carrera de Médico Cirujano

Prof. Carrera Asociado C, TC

liberitzen@yahoo.com.mx

\section{Dr. Noé Contreras González}

Profesor de la carrera de Médico Cirujano

Prof. Carrera Titular A, TC definitivo

noecong10@gmail.com

Carrera Nutriología

Dra. Wendy Daniella Rodríguez García

Profesora de la carrera de Nutriología

Prof. Carrera Asociado C, TC

wen_030@yahoo.com.mx

Mtra. Mariana Isabel Valdés Moreno

Profesora de la carrera de Nutriología

Prof. Carrera Asociado C, TC

nutriologavaldes@hotmail.com
Mtra. Nayeli Anai Vaquero Barbosa

Profesora de la carrera de Nutriología

Prof. Asignatura A

naye7293@gmail.com

Carrera de Psicología

Mtra. Otilia Aurora Ramírez Arellano

Profesora de la carrera de Psicología

Prof. Carrera Asociado C, TC definitiva

otiliaauroraramirezarellano@gmail.com

Dr. José Miguel Sánchez Nieto

Profesor de la carrera de Cirujano Dentista

Prof. Asignatura A

jmsn1904@gmail.com

Carrera de Química Farmacéutico Biológica

M. en C. Taide Laurita Arista Ugalde

Profesora de la carrera de Química Farmacéutico Biológica

Prof. Asignatura A

tdlarista@gmail.com

M. en C. Ana Karen Ruiz Rodríguez

Profesora de la carrera de Química Farmacéutico Biológica

Prof. Asignatura A

hikary1990@gmail.com

M. en C. Jesús Aguilar Castro

Profesor de la carrera de Química Farmacéutico

Biológica

Prof. Asignatura A

profgenclin@gmail.com

Dra. Juana Rosado Pérez

Profesora de la carrera de Química Farmacéutico Biológica

Prof. Carrera Titular A, TC definitiva

juanarosadoperez@gmail.com

Dra. Mirna Ruiz Ramos

Profesora de la carrera de Química Farmacéutico Biológica

Prof. Carrera Asociado C, TC

mirnarr1411@gmail.com 
M. en C. Lizett Castrejón Delgado

Profesora de la carrera de Química Farmacéutico Biológica

Prof. Asignatura A

lizettcastrejon@gmail.com

Dra. Raquel Retana Ugalde

Profesora de la carrera de Química Farmacéutico Biológica

Prof. Carrera Titular A, TC definitiva

retanara@unam.mx

INVESTIGACIÓN Y POSGRADO

\section{Esp. Laura Elena Allende Trejo}

Profesora de la Especialización en Estomatología del Niño y del Adolescente

Prof. Asignatura A

laurallendt@gmail.com

\section{Esp. Citlali Gárate Espinosa}

Profesora de la Especialidad en Estomatología del Niño y del Adolescente

Prof. Asignatura A

garatecitlali9@gmail.com

\section{Mtra. Alicia Arronte Rosales}

Profesora de la Maestría en Neuropsicología

Prof. Asignatura A

arronteposgrado@gmail.com

\section{Lic. Jimena Valeria Aguilar Curiel}

Ayudante de Investigador SNI III

jime_v17@hotmail.com

\section{Lic. Brenda Pedraza Jarquín}

Apoyo en la Unidad de Investigación en Gerontología

Ayudante de Profesor B

brendafeszaragoza@gmail.com

\section{Artículos, materiales aCadémicos, De trabajo y di- VULGACIÓN INCLUIDOS EN LA RAARS}

Artículos publicados disponibles en la página web

- Castrejón-Delgado L, Castelán-Martínez OD, Clark P, Garduño-Espinosa J, Mendoza-Núñez
VM, Sánchez-Rodríguez MA. Effect of tibolone on bone mineral density in postmenopausal women: systematic review and meta-analysis. Biology (Basel). 2021;10(3):211. doi: 10.3390/ biology 10030211.

- García-Martínez BI, Ruiz-Ramos M, Pedraza-Chaverri J, Santiago-Osorio E, MendozaNúñez VM. Hypoglycemic effect of resveratrol: a systematic review and meta-Analysis. Antioxidants (Basel). 2021;10(1):69. doi: 10.3390/antiox10010069.

- Rosado-Pérez J, Castelán-Martínez OD, Mújica-Calderón AJ, Sánchez-Rodríguez MA, Mendoza-Núñez VM. Effect of Tai Chi on Markers of Oxidative Stress: Systematic Review and Meta-Analysis. Int J Environ Res Public Health. 2021;18(7):3458. doi: 10.3390/ijerph18073458.

\section{Materiales de apoyo académico}

- Castelán-Martínez O. La evidencia científica. Boletín de la Evidencia. Casos y Revisiones de Salud. 2019;1(1) Supl 1. Disponible en: https://www.raars.zaragoza.unam.mx/materiales/

- Castelán-Martínez O. EQUATOR Network. Boletín de la Evidencia. Casos y Revisiones de Salud. 2019;1(1) Supl 2. Disponible en: https://www.raars.zaragoza.unam.mx/materiales/

- Mendoza-Núñez VM. Estado del arte del conocimiento. Casos y Revisiones de Salud.2019; 1(1). Disponible en:

https://www.raars.zaragoza.unam.mx/materiales/

- Mendoza-Núñez VM. Casos clínicos: su relevancia en la investigación clínica. Casos y Revisiones de Salud.2019; 1 (2). Disponible en: https://www.raars.zaragoza.unam.mx/materiales/

- Mendoza-Núñez VM. Indización de las revistas científicas. Casos y Revisiones de Salud.2020; 2 (1). Disponible en:

https://www.raars.zaragoza.unam.mx/materiales/

- Sánchez-Rodríguez MA. Estrategias para la búsqueda de información bibliográfica científica 
para una revisión sistemática. Casos y Revisiones de Salud.2020; 2 (2). Disponible en:

https://www.raars.zaragoza.unam.mx/materiales/

- Sánchez-Rodríguez MA. Estrategias para la búsqueda de información bibliográfica científica para una revisión sistemática. Casos y Revisiones de Salud.2020; 2 (2). Disponible en:

https://www.raars.zaragoza.unam.mx/materiales/

- Mendoza-Núñez VM. Factor de impacto e índices H y G. Casos y Revisiones de Salud.2020; 2 (2). Disponible en:

https://www.raars.zaragoza.unam.mx/materiales/

\section{Materiales de trabajo para la elaboración de} UNA TESIS

- Ejemplo Cuadro RS para marco teórico. Disponible en:

https://www.raars.zaragoza.unam.mx/materiales/

- Ejemplo Diagrama PRISMA. Disponible en: https://www.raars.zaragoza.unam.mx/materiales/

- PRISMA 2009 Checklist español. Disponible en:

https://www.raars.zaragoza.unam.mx/materiales/

- Ejemplo Índice. Disponible en:

https://www.raars.zaragoza.unam.mx/materiales/

- Ejemplo de Proyecto RS para registro de TESIS. Disponible en:

https://www.raars.zaragoza.unam.mx/materiales/

- Formato RoB2 Sesgo Ensayos Clínicos. Disponible en:

https://www.raars.zaragoza.unam.mx/materiales/

- Formato Robins-I Sesgo Estudios Cuasi-experimentales Disponible en:

https://www.raars.zaragoza.unam.mx/materiales/.

- Formato Newcastle-Ottawa sesgo para estudios observacionales Disponible en: https:// www.raars.zaragoza.unam.mx/materiales/
- Ejemplo Estimación de la Diferencia de Medias. Disponible en:

https://www.raars.zaragoza.unam.mx/materiales/

\section{INFOGRAFÍAS DE DIVULGACIÓN}

- Conceptos básicos de revisiones sistemáticas. Disponible en:

https://www.raars.zaragoza.unam.mx/materiales/

- Referencia de artículo, estilo Vancouver. Disponible en:

https://www.raars.zaragoza.unam.mx/materiales/

- Referencia de libro, estilo Vancouver. Disponible en:

https://www.raars.zaragoza.unam.mx/materiales/

Finalmente es importante señalar que el contenido de la página web de la RAARS se enriquecerá con el trabajo de todos sus integrantes, por lo que en los próximos meses se incluirá una nueva sección relativa a las tesis de licenciatura y posgrado, realizadas por estudiantes asesorados por alguno de los integrantes de la RAARS con la metodología PRISMA, con el propósito de que sirvan de ejemplo metodológico para otros tesistas. También se actualizarán los cursos y talleres que se impartirán periódicamente dirigidos a profesores y estudiantes de pregrado y posgrado.

\section{AgradeCIMIENTOS}

El manuscrito fue revisado y editado en el Programa para la Investigación Bibliográfica Científica sobre Salud (PIBCIS) de la FES Zaragoza, UNAM. El artículo es parte del material de apoyo didáctico elaborado por la Red Académica Asesora de Revisiones Sistemáticas de la FES Zaragoza, UNAM (RAARS), financiada por la Dirección General de Asuntos del Personal Académico, UNAM, proyecto PAPIME PE203421. Agradecemos a la Mtra. Catalina Armendáriz Beltrán por el diseño de la página Web de la RAARS. 


\section{Referencias}

1. Ferreira González I, Urrútia G, Alonso-Coello P. Systematic reviews and meta-analysis: scientific rationale and interpretation. Rev Esp Cardiol. 2011;64(8):688-696. doi: 10.1016/j.recesp.2011.03.029.

2. Cochrane A. Effectiveness and efficiency. Random reflections on health services. London: Nuffield Provincial Hospital Trust; 1972.

3. Yepes-Nuñez JJ, Urrútia G, Romero-García M, Alonso-Fernández S. Declaración PRISMA 2020: una guía actualizada para la publicación de revisiones sistemáticas. Rev Esp Cardiol. 2021;74(9):790-799. doi: 10.1016/j. rec.2021.07.010.

4. Gerris J. The legacy of Archibald Cochrane: from authority based towards evidence based medicine. Facts Views Vis Obgyn. 2011;3(4):233237.

5. Stavrou A, Challoumas D, Dimitrakakis G. Archibald Cochrane (1909-1988): the father of evidence-based medicine. Interact Cardiovasc Thorac Surg. 2014;18(1):121-4. doi: 10.1093/ icvts/ivt451.

6. Hortiales González AF, Gómez Navarro JA, Barajas Nava LA, Garduño Espinosa J. Archibald Cochrane: evidencia, efectividad y toma de decisiones en salud. Bol Med Hosp Infant Mex. 2017;74(5):319-323. doi: 10.1016/j.bmhi$\mathrm{mx} .2017 .02 .007$.

7. Evidence-Based Medicine Working Group. Evidence-based medicine. A new approach to teaching the practice of medicine. JAMA. 1992;268(17):24205. doi: 10.1001/jama.1992.03490170092032.

8. Rodríguez Campo VA, Paravic Klijn TM. Enfermería basada en la evidencia y gestión del cuidado. Enfermaría Global. 2011;24: 246-253.

9. American Dental Association. Policy on evidence-based dentistry. ADA 2013. Disponible en: https://www.ada.org/en/about-the-ada/adapositions-policies-and-statements/policy-on-evidence-based-dentistry.

10. Daset LR, Cracco C. Psicología basada en la evidencia: algunas cuestiones básicas y una aproximación a través de una revisión bibliográfica sistemática. Ciencias Psicológicas. 2013; VII (2): $209-220$.

11. Del Olmo D, Alcázar V, López del val T. Nutrición basada en la evidencia: presente, limitaciones y futuro. Endocrinol Nutr. 2005;52(Supl 2):2-7.

12. Petticrew M, Roberts H. Systematic reviews in the social sciences. A practicalguide. Oxford: Balckwell Publishing; 2006.

13. Grimán Padua A. Proceso de revisión sistemática de experimentos en ingeniería del software. Tesis Doctoral. Madrid: Universidad Politécnica de Madrid, Facultad de Informática; 2017.

14. De la Cruz-Cardoso D, Mendoza-Núñez VM. Manual para la Elaboración de Proyectos de Investigación en Ciencias de la Salud. México: FES “Zaragoza”, UNAM; 1989.

15. Mendoza-Núñez VM, Romo-Pinales R, Sánchez-Rodríguez M, Hernández- Zavala S. Investigación. Introducción a la metodología. México: FES “Zaragoza”, UNAM; 1997.

16. Mendoza-Núñez VM, Sánchez-Rodríguez MA. Análisis y difusión de resultados científicos. México: FES "Zaragoza", UNAM; 2001.

17. Sánchez Rodríguez MA, Rosas Barrientos V, Mendoza-Núñez VM. Metodología de la investigación. Un enfoque práctico. México: FES Zaragoza, UNAM; 2018. Disponible en: https://www. zaragoza.unam.mx/area-quimico-biologicas/

18. Facultad de Estudios Superiores Zaragoza, UNAM. Red Académica Asesora de Revisiones Sistemáticas (RAARS). México: FES Zaragoza, UNAM;2021. Disponible en: https://www.raars.zaragoza.unam.mx/ [Accesada 29 de octubre, 2021] 